\title{
Profesor Investigador y Formación de Profesores
}

Teacher as Researcher and Teacher Education

Alberto Villani ${ }^{-1}$ y Verónica Guridi ${ }^{2}$

IF- Universidade de São Paulo ${ }^{1}$ Con apoyo parcial del CNPq ${ }^{1}$. EACH - Universidade de São Paulo ${ }^{2}$ avillani@if.usp.br, veguridi@usp.br

\section{Resumen}

En este trabajo realizamos una revisión histórica sobre el concepto de "profesor investigador", rescatando la obra de autores como Stenhouse, Elliott y Schön, entre otros. También presentamos una síntesis de algunas discusiones actuales realizadas en la literatura, en las que se enfatiza la necesidad de aproximar el universo del profesor con el de la investigación académica, de modo a establecer una colaboración productiva entre investigadores académicos y profesores. La revisión es complementada con los resultados de nuestras investigaciones sobre las condiciones para la indusión de las investigaciones del profesor en la academia y algunas implicaciones para la formación de profesores.

\section{Palabras clave}

Formación de profesores; relación docencia-investigación; profesor investigador; revisión histórica.

\section{Abstract}

In this work, we present an historical review of the concept "teacher as researcher", based on the contributions of some authors like Dewey, Stenhouse, Elliott, Schön. We also make a synthesis of some recent discussions developed in literature, in which it is emphasized the necessity to approximate the teacher's universe to those of academic research, in order to establish a genuine collaboration between academics and teachers. The review is completed with the results of our researches about the conditions for the inclusion of teacher's research in the academic world and some implications for teacher education.

\section{Keywords}

Teacher education; teaching-research relationship; teacher as researcher; historical review.

\section{Introducción}

La indusión de profesores de enseñanza básica en el universo de la investigación siempre fue y aún sigue siendo un tema controvertido. Por un lado, se reconoce la importancia de la investigación para el profesor que trabaja en la escuela pero, al mismo tiempo, se imponen ciertos padrones que restringen el tipo de investigación que ese profesor puede realizar. El primer aspecto parece estar relacionado al reconocimiento del saber del profesor como fuente de datos para una investigación: el profesor es portador de un saber, construido en la interacción con la práctica. El segundo aspecto tiene que ver con las restricciones que son impuestas a ese tipo de investigaciones. ¿Será que pueden considerarse investigaciones "cient́ficas"? Algunos defienden que las actividades de enseñanza exigen habilidades diferentes de la actividad de investigación y por ello la formación de profesores debería estar volcada para el desarrollo de competencias exigidas para sus funciones específicas. 
En este trabajo intentaremos contribuir para esdarecer ese problema de dos maneras. Por un lado, procuraremos sintetizar las razones que aparecieron en los trabajos de los grandes autores que abordaron el tema en el pasado y recientemente. Complementaremos esa revisión con algunos resultados de nuestras investigaciones más recientes, centrándonos principalmente en las contribuciones que la Universidad puede brindar para que los profesores de Ciencias se transformen en investigadores. Conduiremos con algunas consideraciones sobre la formación de profesores de Ciencias para la investigación.

\section{Revisando los Clásicos}

El marco inicial de esa tendencia debe ser localizado en la Filosofía Educacional de Dewey. Rompiendo con la tradición conservadora, Dewey declaró que la educación no tenía el papel de conservar lo establecido, sino de promover lo nuevo, a partir de una práctica democrática y con el privilegio de la experiencia, entendida como capaz de colocar al aprendiz en un nuevo lugar con relación al medio en el que actúa. Con ello, pretendía superar el abordaje excesivamente técnico que caracterizaba la actividad de enseñanza de los profesores en el aula, como el ejercicio pedagógico para la transmisión de conocimientos definitivos. Por el contrario, para Dewey, una idea debe pasar por el veredicto de la experiencia, promoviendo nuevas experiencias, para ser asimilada como verdadera. Un corolario de esa visión de la educación fue el foco en la reflexión como estrategia necesaria para enfrentar los desafíos encontrados en el proceso de transformación de los aprendices en miembros de una sociedad democrática. Para Dewey, el acto reflexivo del profesor competente debería acompañar su práctica, rompiendo con la toma de decisiones rutinarias, que no podrían considerar las novedades involucradas en la experiencia de los aprendices. Dewey influenció profundamente la educación en su país, durante por lo menos medio siglo y también fue reconocido en otros países.

En Brasil, en partiaular Anísio Teixeira, desarrolló su práctica político educacional con la inspiración del pensamiento de Dewey, con quien comulgaba profundamente, a pesar del dima político bastante distante de la democracia americana. Según Anísio, para la superación de la tradición colonial brasilera y el florecimiento de una sociedad democrática se precisaba un compromiso sistemático de los profesores en la utilización de la reflexión para enfrentar los desafíos y la osadía para proponer soluciones. La propuesta de Paulo Freire, en las décadas de sesenta y setenta, constituyó un avance y una continuidad de los legados de Anísio Teixeira, especialmente al focalizar la relación dialógica entre docente y alumno y el compromiso con una búsqueda de una educación libertadora para ambos. Sin dudas, a pesar de no utilizar el nombre de profesor investigador de su práctica, Freire consideraba esencial que el profesor encontrase las mejores formas y contextos para que los alumnos pudieran ejercer su ciudadanía. Sus ideas tuvieron tránsito en Brasil y en el exterior, a pesar de las condiciones adversas de un país inmerso en una dictadura militar hasta la mitad de la década de 80 . En síntesis, la reflexión y la investigación del profesor estuvieron caracterizadas en esta primera fase como instrumentos para conferir a la educación una connotación civilizadora y libertadora. Los herederos más completos de este enfoque fueron los investigadores y educadores que se dedicaron al movimiento Ciencia, Tecnología y Sociedad (CTS), en el inicio de la década de 70 y posteriormente, porque además de orientar su actuación y reflexión en la dirección de una educación civilizadora, eligieron como contenido objetivo la contribución de la ciencia y de la tecnología para la democratización de la sociedad y para el avance de los derechos humanos.

El foco de las consideraciones parece haberse desplazado en parte con las propuestas de Stenhouse. En la década de 70, en Inglatenra, él estaba preocupado con la emancipación del profesor, para la superación del paternalismo y de la dependencia que caracterizaban la actuación docente. Aún en los años 60, la investigación apareció por primera vez con este autor, como 
soporte para la autonomía del profesor, focalizando principalmente el currículo, o sea, el proceso de transmisión del conocimiento en el aula (Stenhouse, 1975). Al fundar el CARE (Center for Applied Research in Education), Stenhouse pretendía democratizar la investigación educativa, involucrando a los profesores en su realización y al mismo tiempo tomando sus resultados capaces de ayudarlos en su desenvolvimiento profesional. Consiguió juntar profesores e investigadores académicos, transformando la investigación la base de la enseñanza y de la autogestión de la práctica. 0 sea, la investigación era vista como el fundamento de la emancipación del profesor, en cuanto era capaz de producir nuevos conocimientos ligados a la práctica docente. Esa tesis fue retomada por Elliott, su sucesor después de su muerte prematura en 1982. La situación generalizada que Elliott quería modificar era la falta de entusiasmo y la resistencia de los profesores a las investigaciones académicas en Educación y, por otro lado, la desvalorización de su práctica por parte de los investigadores, que frecuentemente comparaban negativamente las actividades del aula con sus modelos ideales. La idea del profesor investigador de su práctica docente (Elliott, 1986) fue ampliada, intentando colocar el foco en dos efectos complementarios: por un lado, enfrentar la creciente complejidad del aula, que involucraba también el propio papel del profesor en el aula y, por otro, realizar un análisis más apropiado de las dificultades y de los problemas de la enseñanza, tornándose una alternativa a los trabajos académicos mucho más dependientes de las teorías y de los modelos vigentes. En el caso espeć́fico de la educación en ciencias, la década de 80 fue dominada por el movimiento de las Concepciones Altemativas y por el modelo de Cambio Conceptual. De esa manera, varios profesores, principalmente en la enseñanza superior y media, se dedicaron a investigar los efectos de la focalización explícita de las concepciones de los alumnos en sus prácticas docentes.

El rescate del status del profesor de enseñanza básica por medio de la valorización de la reflexión y de la investigación fue también el lema de Schön (1983), que retomó y especificó la propuesta de Dewey sobre la reflexión, casi identificando el profesor reflexivo con el profesor investigador. Para él, la reflexión del profesor sobre su práctica puede ser realizada en varias fases: i) refiexión en la acción, referida a los procesos de pensamiento que se realizan durante el desenvolvimiento de la experiencia de enseñanza y aprendizaje, con el objetivo de identificar los problemas que surgen durante la acción y proponer cambios en el transcurso de la intervención; ii) reflexión sobre la acción: ocurre en un momento posterior a la intervención y está orientada a repensar la acción, describiendo y objetivando lo ocurrido; iii) reflexión sobre la reflexión en la acción implica un distanciamiento mayor de la acción y la interpretación del propio proceso, posibilitando una reflexión continua de la práctica.

Las ideas de Schön tuvieron mucho éxito, especialmente porque permitieron múltiples abordajes, articulaciones e investigaciones colaborativas, siendo útiles tanto en la planificación como en el análisis de breves procesos de actualización de los profesores, incentivados a romper la rutina tanto por medio de una reflexión atenta durante y después de sus dases, como de largas investigaciones en colaboración que aborden el proceso docente a partir de referenciales apropiados. En la misma década, Carr y Kemmis (1988) elaboraron un análisis crítico de la situación educacional de la época, dominada por dos movimientos que, de alguna manera, marcaban la sumisión de la educación a las otras disciplinas. Por un lado, el dominio del enfoque tecnocrático, que consideraba a la educación como medio para "producir la conformidad con una imagen de persona educada ajustada de antermano" (op. cit, p. 32). Por otro, el enfoque fundamentalista consideraba la educación como un apéndice de otras disciplinas (psicología, sociología, historia y filosofía), que sustentaban su aparato teórico y justificaban sus principios educativos, sin ninguna referencia a las prácticas educativas. La propuesta de los autores fue la promoción de la investigación acción - en el sentido emancipatorio, tanto de la práctica educativa como del saber del educador.

En el caso de la enseñanza de ciencias, las ideas de Schön comenzaron a encontrar aplicación en el final de la década de $\mathbf{8 0}$, por la crisis del Modelo de Cambio Conceptual, que obligó a los

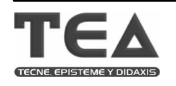


investigadores y profesores a una reflexión más profunda sobre la práctica docente. No habiendo más un guía seguro que los orientase y, al mismo tiempo, comenzando a percibir un desinterés de los alumnos con relación a la ciencia, varios profesores desplazaron la reflexión sobre su práctica docente hacia la búsqueda de nuevas motivaciones. Meta-cognición, problemas en abierto, método de proyectos, entre otros, fueron los nuevos instrumentos en la enseñanza de ciencias y en las correspondientes investigaciones: tales metodologías exigían una gran flexibilidad del profesor y se acoplaban de manera resonante con los diversos tipos de reflexión propuestos por Schön.

\section{Algunas discusiones actuales}

Las discusiones actuales focalizan las diferentes características de la práctica académica y de la actividad docente e intentan establecer algunos caminos posibles de superación de las barreras entre esos dos universos.

En la década de 90, varios autores trabajaron sobre la relación del profesor con la investigación, principalmente Nóvoa, Perrenoud, Tardiff y Zeichner. Entre ellos, quien más se destacó por sus contribuciones con respecto a la participación de los profesores de enseñanza básica en la investigación educacional $y$ al reconocimiento de la investigación-acción como legítima en la Universidad, fue Zeichner. Para ese autor, existe un problema de mutua falta de entendimiento entre profesores e investigadores. 0 sea, cada uno de los grupos considera irrelevantes las preguntas de investigación del otro. Él llama la atención para el hecho de que, a pesar de la revolución causada por el concepto de 'profesor investigador', son los propios profesores los que no se reconocen como productores de conocimiento, delegando esa tarea en los investigadores externos (Zeichner, 1995).

El autor destaca varias razones que llevaron al alejamiento de los profesores de la investigación académica: el lenguaje excesivamente técnico utilizado en las publicaciones; el hecho de que los profesores se ven retratados de forma negativa en la mayoría de las investigaciones académicas, viendo a los investigadores como personas insensibles e incapaces de comprender sus verdaderas condiciones de trabajo; la relación de poder establecida entre ambos grupos (los profesores se sienten explotados por los investigadores, utilizados apenas como instrumentos en las investigaciones).

Esa situación torma difícil la relación entre ambos grupos y, por lo tanto, la transferencia de resultados de investigaciones en el ámbito escolar, así como el aprovechamiento de los saberes de los profesores en el ámbito académico. Zeichner denuncia la falta de esfuerzo realizado por la Universidad para cambiar la situación antes descripta. Propone la investigación colaborativa para superar la división entre profesores y académicos. Sugiere tres estrategias para romper con esa separación: a) involucrar a los profesores de las escuelas en discusiones sobre el significado y la importancia de las investigaciones académicas; b) desarrollar proyectos de investigación en colaboración con los profesores de escuela, en los que los viejos modelos jerárquicos sean superados y c) apoyar proyectos de investigación-acción desarrollados por los educadores, aceptando legítimamente el conocimiento producido en ese proceso. El objetivo de él es formar profesores como pensadores autónomos y prácticos reflexivos que se comprometan con la calidad de la educación para todos los alumnos. En ese contexto, la investigación-acción aparece como el camino metodológico más adecuado para alcanzar tal propósito.

Durand et al. (2005), caracterizan la epistemología de los saberes y la epistemología de la acción y consideran que existe una división entre ellas. La epistemología de los saberes realiza una dara distinción entre investigación y formación docente, y una separación entre la fase de investigación y la formación. Investigadores y profesores no interaccionan strictu sensu, ya que la prevalencia de citerios cientúficos exduye la posibilidad de que los formadores y los profesores 
trabajen de igual a igual. Profesores son solamente sujetos de investigación y se busca producir conocimientos universales. Por otro lado, en la epistemología de la acción existe una permanente asociación entre ambas y la subjetividad de los actores es lo más importante; los estudios son contextualizados y procuran comprender la singularidad de las situaciones estudiadas. También hay una participación colectiva de diferentes atores (investigadores, profesores, formadores...): los profesionales de la red escolar tienen un papel primordial durante el proceso de investigación, desde la formulación de las preguntas y de los objetos de estudio hasta la elaboración de condusiones, porque se busca tanto la producción de conocimientos como la transformación de las situaciones.

Los autores destacan que, a pesar de esa oposición aparente, ambas epistemologías pueden complementarse e integrarse en una propuesta de formación que una los hilos entre investigaciónacción-intervención de una manera dinámica y que contemple los criterios de rigor y pertinencia. Para ello, proponen un programa de ergonomía/formación que tome visible el trabajo de profesores y formadores, así como su actividad, buscando la comprensión y transformación de esa actividad. El programa pretende ser un auxiliar en la formación docente, al mismo tiempo en que busca producir conocimientos.

Lüdke y Cruz (2005) discuten la relación entre la investigación y el profesor de educación básica, a partir de datos de un programa integrado de investigación sobre el tema. Como una forma de superación de la barrera entre teoría y práctica, proponen una formación docente en la que teoría y práctica estén ligadas. Las autoras conduyen que la formación para la investigación tiende a generar en los profesores representaciones vinculadas a un formato de investigación académica. Esas representaciones no dejan espacio para el desarrollo de visiones paralelas, más amplias, volcadas para el trabajo en las escuelas, sin por eso descuidar el rigor de toda investigación. Según las autoras, es preciso ampliar el concepto tradicional de 'investigación', para que puedan ser induidos otros diseños más próximos de aquellos que el profesor de enseñanza básica puede desenvolver. Aún existe una cuestión que continúa bastante oscura: la propia identidad de la investigación desarrollada por el profesor de enseñanza básica. La investigación-acción o la investigación colaborativa han sido consideradas opciones metodológicas para que el profesor pueda desarrollar una actividad de investigación. Sin embargo, las autoras alertan que ese tipo de investigación no es bien recibido en el medio académico.

Tardiff y Zourhlal (2005), argumentan que existe un "pozo" profundo entre los investigadores y los profesores, debido a un problema de comunicación, caracterizado por universos discursivos diferentes: investigadores y profesores no hablan la misma lengua. Para ellos, los investigadores deben aprender a popularizar su discurso, traduciéndolo en un lenguaje menos hermético y preparar mejor a los profesores para la lectura y decodificación de los discursos de la investigación. En ese sentido, hay que reformular los programas de formación inicial y realizar investigaciones en colaboración, formuladas y ligadas a problemas generados en las escuelas. Ese tipo de investigaciones debería ser reconocido en el ámbito académico como un tipo legítimo de investigación y producción de conocimiento. Para resolver el problema comunicativo, por lo menos en parte, sería necesario diversificar la carrera docente. De esa forma, una parte de la profesión docente debería ser reconocida en función de la inversión del profesional en la dífusión y participación en investigaciones. Esto ayudaría a resolver también el problema del tiempo, permitiendo que los profesores dediquen más tiempo a las actividades de investigación.

En síntesis, podemos resumir la evolución de los desdoblamientos de la propuesta de transformar al profesor en un profesional marcado por la reflexión y por la investigación sobre su práctica, por medio de varias fases. En una primera fase, la refiexión del profesor aparece vinculada a la posibilidad de construcción de una sociedad más justa, democrática en la cual el papel de la reflexión del profesor es elemento central para esa transformación. En seguida, la reflexión aparece vinculada a los procesos de investigación sobre la práctica, con el objetivo de tomarla más efectiva 
para la democratización de la enseñanza. Finalmente, en los días actuales, hay una fase en la que la investigación del profesor es articulada a aquella realizada en la Universidad, integrándose en proyectos de colaboración, en los cuales las antiguas posiciones jerárquicas sean superadas.

\section{Nuestra contribución}

Nuestra contribución estuvo orientada por la investigación acerca de las posibilidades de cooperación entre la Universidad y los docentes, facilitando la tarea de que éstos se transformen en investigadores. ¿Cuáles son los caminos que un profesor puede transitar de manera efectiva para ser reconocido como investigador y cuáles son las condiciones ofrecidas por la Universidad para la realización de esa tarea? Para ello, Guridi (2007) realizó un estudio con profesores de enseñanza básica matriculados en una disciplina sobre introducción a la investigación en Enseñanza de Ciencias. La autora estudió el proceso de iniciación en la investigación en los casos de profesores que deseaban investigar su propia práctica. Realizando una colecta de datos durante un semestre en el contexto de esa disciplina, montó un esquema para comprender el proceso de indusión de esos profesores en el universo de la investigación. Analizó de forma detallada la evolución de las ideas y de los proyectos de investigación de seis profesores con diferentes históricos escolares y las actividades propuestas por el responsable de la disciplina. Como resultado, fue considerado que la inclusión era exitosa cuando existía un movimiento de "ida y vuelta": por un lado, el profesor realiza un esfuerzo para aproximarse a los cánones de la Academia, incorporando progresivamente criterios y herramientas de la comunidad científica y, por otro, esta última realiza esfuerzos en el sentido de flexibilizar ciertos criterios tradicionales sobre lo que significa "hacer investigación", para que el profesor sea considerado como un igual en ese universo y pueda explorar los saberes de su práctica.

La tabla 1 muestra un cuadro donde puede observarse ese proceso de "ida y vuelta", con indicadores de los movimientos de inclusión de ambas partes. El cuadro, que incorpora varias de las propuestas de los otros autores comentados aquí, ha sido útil para caracterizar la forma en que se produce la iniciación en la investigación en el caso de profesores de enseñanza básica que pretenden realizar investigaciones que sean reconocidas en el ámbito académico.

\section{Indicadores del movimiento de inclusión} del profesor en el medio académico

Lenguaje utilizado (esfuerzo de aproximación con el lenguaje y cultura de la Academia); Incorporación de herramientas y criterios de la comunidad (identíficación de las fases de una investigación sistemática);

Elaboración de relatos de actividades consistentes y articulados (explicitación de los elementos fundamentales de una investigación en las actividades realizadas);

Explicitación de posición crítica en las actividades realizadas en la disciplina;

Explicitación de contribuciones provenientes de la propia práctica para la investigación (reconociendo la complejidad de la práctica); Reconocimiento del tipo de conocimiento a ser producido (intervención o investigación).
Indicadores del movimiento de inclusión realizado por la academia

Facilitación de la comunicación (esfuerzo por traducir el lenguaje científico para uno más familiar al profesor y esfuerzo para interpretar su lenguaje en lenguaje científico);

Flexibilización de los criterios de selección del objeto de estudio (aceptación de la investig. sobre la propia práctica y esfuerzo para tornarla consistente como objeto de estudio);

Flexibilización de criterios sobre metodología de investig. (aceptación de metodologías adaptadas a las condiciones de trabajo en el aula);

Reconocimiento $y / 0$ valorización de los conocimientos prácticos del profesor (con intervenciones que consideren lo que ese profesor informa a partir de sus saberes);

Reconocimiento de las contribuciones específicas del profesor (focalizando las informaciones que solamente él puede brindar).

Tabla No. 1. Indicadores de los movimientos de indusión realizados por el profesor y por la Academia (Guridi, 2007) 
En las condusiones, se resalta que los elementos con mayor influencia positiva en el proceso de indusión están relacionados con los esfuerzos de traducción entre lenguajes y con la flexibilización de criterios sobre metodología de investigación. Cabe mencionar aquí que flexibilizar criterios no significa restar rigor a la investigación, sino de tornarla rigurosa de acuerdo con otros criterios, más próximos de la práctica cotidiana del profesor, sin por ello dejar de lado aspectos dave vinculados al rigor necesario en todo tipo de investigación. Se trata de valorizar aquello que solamente el profesor puede brindar, para incorporarlo funcionalmente en la realización de una investigación consistente.

En consecuencia, el problema ahora consiste en definir lo que significa "investigación": la indusión de los profesores de enseñanza básica en el mundo de la investigación requiere primero que se esclarezca el significado del término "investigación" en la formación de profesores y, principalmente, que se defina auál es el tipo de investigación que ese profesor estaría en condiciones de desenvolver.

Otra investigación, aún en desarrollo, intentó localizar las caractenísticas de los profesores de enseñanza media que consiguieron conducir hasta el final investigaciones referentes a su práctica docente y principalmente, reauperar las motivaciones que los sostuvieron en la superación de las dificultades encontradas. En esos casos, entrevistas y análisis de los diarios de investigación y de los trabajos producidos por los profesores, se constituyen en las fuentes más importantes de informaciones. Fueron analizados auatro casos, arrojando los resultados que discutiremos en los párrafos siguientes.

A pesar de no presentar un éxito extraordinario, desde el punto de vista metodológico o didáctico, las investigaciones posibilitaron el descubrimiento de fallas en la conducción docente y un cambio subjetivo de los investigadores con relación a la actividad de enseñar ciencias. En particular, una conquista común fue la disminución de la importancia de algunas creencias 0 ansiedades que anteriormente distorsionaban su mirada de profesores: querer agradar a todos, confiar demasiado en la planificación, evitar sistemáticamente el confilicto de los alumnos, ser perfecto en la actuación. En general, además de ser reconocidas explícitamente, esas creencias fueron enfrentadas por los profesores, reduciendo de una manera efectiva sus influencias en la práctica docente, al retomar su actuación después de la experiencia. En cuanto a la motivación para querer investigar sobre su práctica en un curso de postgrado, inicialmente hubo una insatisfacción de los profesores con relación al bajo interés de los alumnos, al escaso avance cognitivo y a la dificultad de inclusión de alumnos marginados. Esa insatisfacción se convirtió en el motor para que estos profesores decidieran poner a prueba una propuesta didáctica innovadora, junto con la perspectiva, más 0 menos consciente, de que el post-grado ampliaría las posibilidades docentes, eventualmente en el nivel superior de enseñanza o en otros campos que les dieran mayor satisfacción. Para algunos, también existía el desafío de producir nuevos conocimientos que podrían ser compartidos con otros profesores.

Entre los obstáculos para la conducción de la investigación podemos citar: la escasez de tiempo disponible para la investigación, las resistencias intemas para poner en juego la subjetividad, los esfuerzos para conseguir un lugar para la realización de la experiencia, la búsqueda de interlocutores en la Academia, los riesgos de que la experiencia no llegue hasta el final, la poca visibilidad de los resultados de la experiencia y los conflictos inherentes a la interpretación de los resultados. Un elemento significativo, reconocido como importante para superar esos obstáculos, fue la elaboración de un diario de investigación como ocasión de reflexión inicial sobre su práctica y también como registro fundamental para la obtención de los datos de la investigación y para las discusiones con los interlocutores privilegiados. Sin embargo, el desplazamiento del foco de la investigación para el análisis fue reforzado por las posibilidades de registros objetivos sobre su práctica docente, como las grabaciones en video, las anotaciones de los observadores y sus comentarios, que ayudaron a que los profesores se distancien de su práctica para enfrentarla con 
otra mirada. Para los cuatro profesores, el encuentro con categorías relativas a la subjetividad, ayudó a lidiar de manera segura con la problemática revelada por los diarios, por las sugerencias de los interlocutores o por la relación con los alumnos. También fue muy importante el auxilio de grupos de interlocutores en la Academia, formado por colegas y directores de tesis, que contribuyeron con sugerencias y con nuevos puntos de vista en el análisis de las auestiones presentadas y en la sustentación de la metodología de investigación. Esos grupos ayudaron a los profesores a percibir la novedad de sus contribuciones y a presentar sus trabajos en Congresos. Eso entró en resonancia con la persistencia de los profesores como investigadores y con la apertura de nuevas posibilidades académicas.

\section{Conclusiones y comentarios finales}

Para Ponte (2005), la investigación sobre la propia práctica es diferente de la investigación académica, ya que los propósitos son diferentes y, por lo tanto, los citerios de evaluación son también diferentes. Una herramienta fundamental en el proceso de investigación sobre la propia práctica es la reflexión del profesor, que puede ser realizada mediante varios diseños metodológicos, no sólo la investigación acción. Lo esencial es que los profesores construyan conocimientos analizando situaciones reales, recogiendo datos de sus prácticas y reflexionando sobre ellos con el apoyo de otros (profesores y/o investigadores). El proceso fundamental de distanciamiento puede ser obtenido por medio de varias estrategias: usando lentes analíticas, focalizando el objeto (por ejemplo, el discurso de los alumnos, la actividad de los alumnos, el trabajo en grupo), generando varios tipos de registros de la práctica (video, audio, registros escritos...) y reflexionando con otros (profesores con preocupaciones y problemas semejantes, con una experiencia profesional diferente, con investigadores de enseñanza superior) y comunicando los resultados en encuentros de investigación y encuentros de profesionales.

Análogamente, André (2001) considera como condiciones mínimas para tornar un profesor investigador: disposición para investigar y cuestionar su práctica; formación para aprender a formular problemas y cuestiones investigativas, esfuerzo para conocer y seleccionar métodos e instrumentos de observación y análisis. También parece relevante tener tiempo para participar de grupos de estudio en su lugar de trabajo, conseguir espacio para realizar investigación y tener acceso a materiales, fuentes de consulta y bibliografía especializada.

Nuestras condusiones parecen estar muy próximas a las de estos autores. La novedad presentada aquí se refiere al enfoque que privilegió la articulación de las motivaciones de los profesores que sustentaron las diferentes etapas del proceso y posibilitaron la superación de los conflictos y de las dificultades encontradas progresivamente por ellos durante la investigación. Nuestro trabajo parece relevante por brindar informaciones acerca de los casos en que el profesor investiga sobre la propia práctica. Estamos sugiriendo que los auríaulos brinden la posibilidad de que los formadores monitoreen las prácticas de investigación realizadas por los profesores aprendices. O sea, parece no ser suficiente disautir referenciales, enseñar procedimientos metodológicos, analizar técnicas de investigación y realizar ensayos prácticos de investigación para que el profesor consiga conduir una investigación. Es necesario también tomar cuidado para que el profesor consiga concluir una investigación. Es preciso también que él aprenda a lidiar con las dificultades, localizando los conflictos y procurando soluciones, perspectivas 0 ayudas, que tomen esos obstáculos transponibles. El enemigo del profesor que investiga su práctica es el abandono del esfuerzo por falta de intermediarios. A pesar de no haber una regla que pueda evitar esta situación, el conocimiento de cómo ella se presenta y de cómo fue enfrentada por otros, puede ayudar a encontrar la solución. 


\section{Bibliografía}

André, M. (2001). Pesquisa, Formação e Prática Docente. In: André, M. (Org.) $O$ papel da pesquisa na formação e na prática dos professores. Campinas: Papirus.

Carr, W. \& Kemmis, S. (1988). Teoria Crítica de la Ensenanza; la investigación-acción en la formação del professorado. Barcelona: Martinez Rocha.

Durand, M., Saury, J. y Veyrunes, P. (2005). Relações fecundas entre pesquisa e formação docente: elementos para um programa. Cademos de pesquisa, v. 35 (125), pp. 37-62

Elliott, J. (1986). La investigación-acción en el aula. Valencia, Generalita Valencia. 1986.

Guridi, V. (2007). A indusão do professor de ensino básico na pesquisa: um desafio institucional. Tesis (Doctorado en Educación). Facultad de Educación. Universidad de San Pablo. San Pablo. 204 p.

Lüdke, M. y Cruz, G Barreto da. (2005). Aproximando universidade e escola de educação básica pela pesquisa. Cademos de pesquisa, v. 35 (125), pp. 81-109.

Ponte, J. P. 0 interacionismo simbólico e a pesquisa sobre a própria prática. Revista Pesquisa Qualitativa, 1, 107-134, 2005.

Schön D. (1983). The Reflective Practicioner. New York: Basic Books.

Stenhouse, L (1968). The humanities curriaulum project. Joumal Of Curriaulum Studies, v. 1(1), pp. 26-33.

Tardiff, M. y Zourhlal, A. (2005). Difusão da pesquisa educacional entre profissionais do ensino e árculos acadêmicos. Cadernos de pesquisa, v. 35 (125), pp. 13-35.

Zeichner, K. (1995). Reflections of a teacher educator working for social change. En: Korthagen, F. and Russel, T. (Eds.) Teachers who teach: reflections on teacher education (pp. 11-24). Londres: Falmer press. 